\title{
Can Good Infection Control Be Obtained in One-stage Exchange of the Infected TKA to a Rotating Hinge Design? 10-year Results
}

\author{
Akos Zahar MD, Daniel O. Kendoff MD, PhD, Till O. Klatte MD, \\ Thorsten A. Gehrke MD
}

Published online: 23 June 2015

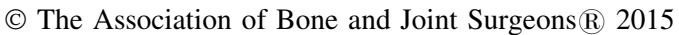

\begin{abstract}
Background Prosthetic joint infection (PJI) occurs in $1 \%$ to $2 \%$ of total knee arthroplasties (TKAs). Although two-stage exchange is the preferred management method of patients with chronic PJI in TKA in North America, one-stage exchange is an alternative treatment method, but long-term studies of this approach have not been conducted.

Questions/purposes We reviewed our minimum 9-year results of 70 patients who underwent one-stage exchange arthroplasty with a rotating hinge design to determine: (1) What was the proportion of patients free of infection? (2)
\end{abstract}

Each author certifies that he or she, or a member of his or her immediate family, has no funding or commercial associations (eg, consultancies, stock ownership, equity interest, patent/licensing arrangements, etc) that might pose a conflict of interest in connection with the submitted article.

All ICMJE Conflict of Interest Forms for authors and Clinical Orthopaedics and Related Research ${ }^{\mathbb{R}}$ editors and board members are on file with the publication and can be viewed on request.

Clinical Orthopaedics and Related Research ${ }^{\circledR}$ neither advocates nor endorses the use of any treatment, drug, or device. Readers are encouraged to always seek additional information, including FDA approval status, of any drug or device before clinical use.

Each author certifies that his or her institution approved the human protocol for this investigation, that all investigations were conducted in conformity with ethical principles of research, and that informed consent for participation in the study was obtained.

This work was performed at Helios ENDO Klinik, Hamburg, Germany.

\section{A. Zahar ( $\square)$, D. O. Kendoff, T. A. Gehrke}

Center for Septic Surgery, Helios ENDO Klinik, Holstenstr. 2, 22767 Hamburg, Germany

e-mail: akos.zahar@helios-kliniken.de

T. O. Klatte

Department of Trauma-, Hand- and Reconstructive Surgery, University Medical Center Hamburg-Eppendorf, Hamburg, Germany
What was the patient rate of survival free of any reoperation? (3) What were the clinical outcomes as measured by Hospital for Special Surgery scores? (4) What proportion of patients developed radiographic evidence of loosening?

Methods All one-stage revision TKAs for infection between January 1 and December 31, 2002, with a minimum 9-year followup (mean, 10 years; range, 9-11 years), in which patients had been seen within the last 1 year, were included in this retrospective review. During that period, 11 patients with infected TKAs were treated with other approaches (including two-stage approaches in eight); the general indication for one-stage revision was the diagnosis of PJI with a known causative organism. Exclusion criteria were culture-negative preoperative aspiration, known allergy to local antibiotics or bone cement, or cases in which radical débridement was impossible as a result of the involvement of important anatomical structures. Eightyone patients with PJI were seen during this period; 70 underwent one-stage exchange using our strict protocol and were reimplanted with a rotating hinge TKA. Eleven patients $(15.7 \%)$ were lost to followup. Hospital for Special Surgery scores were recorded and all radiographs were evaluated for prosthetic loosening. Failure was defined as revision surgery for infection or any other cause.

Results Our 10-year infection-free survival was 93\% (mean, $4.1 ; 95 \%$ confidence interval $[\mathrm{CI}], 89 \%-96 \% ; \mathrm{p}<0.007)$; and the patient 10-year survival rate free of revision for other causes was $91 \%$ (mean, 5.2; 95\% CI, 86\%-95\%; p < 0.002). Mean Hospital for Special Surgery knee score at last followup was $69.6( \pm 22.5 \mathrm{SD}$; range, 22-100) and the mean improvement in Hospital for Special Surgery knee score from preoperative to most recent followup was 35 ( $\pm 24.2 \mathrm{SD}$; range, 13-99). Evidence of radiographic loosening was seen in 11 patients at last followup, whereby in six patients, there was need for revision surgery. 
Conclusions Our study results showed an overall infection control rate of $93 \%$ and good clinical results using our one-stage approach, which combines aggressive débridement of the collateral ligaments and posterior capsule with a rotating hinge implant. These results are comparable with two-stage techniques at a followup of 10 years; further research into one-stage exchange techniques for PJI in TKA appears warranted.

Level of Evidence Level IV, therapeutic study.

\section{Introduction}

Although most patients with prosthetic joint infection (PJI) after TKA are treated with a two-stage exchange arthroplasty $[15,19,24,27,30,32,35]$, the one-stage approach has been carried out by certain institutions around the world since the 1970s $[8,10,41]$.

Although most series report an infection control rate of approximately $90 \%$ for two-stage exchange arthroplasty, it has been shown that the general risk of patient mortality is increased by PJI and its surgical treatment [44], and a twostage approach might be associated with an even higher mortality risk, at least in THA [3]. Some studies have shown possible advantages of one-stage exchange for the infected TKA in selected patients [14], including the need for only one surgical procedure, shorter hospitalization time, reduced time on antibiotic therapy, reduced overall costs, and improved patient satisfaction [16, 29, 39, 40]. In addition, an increasing number of publications have reported on one-stage exchange in TKA and THA within the last few years, showing high success rates $[14,20,36,39,40]$. However, these publications share certain limitations: small numbers of patients $(<20)$; short followup ( $<5$ years); and strict exclusion criteria such as multiresistant organism-based infections, reinfection, significant bone loss, relevant comorbidities, sinus tract, and other relevant soft tissue complications [26]. Thus, a direct comparison of success rates with the more often used two-staged approaches cannot be easily drawn, and interpretation of the literature becomes complex $[19,32]$.

Therefore, we reviewed the minimum 9-year results of our 70 patients who underwent one-stage exchange arthroplasty with a rotating hinge design to: determine (1) survival free of infection; (2) survival free of any reoperation; (3) clinical outcome as measured by Hospital for Special surgery (HSS) score; and (4) radiographic outcome as determined by radiographic evidence of prosthetic loosening.

\section{Patients and Methods}

After approval of the local ethical committee, a retrospective analysis of data collected from the ENDO Klinik,
Hamburg, Germany, was performed in all patients who underwent a one-stage exchange arthroplasty for infected TKA between January 1 and December 31, 2002.

Seventy patients with one-stage septic TKA revision were included in our review and 11 patients with other than one-stage procedures were excluded. We excluded from the study all patients who underwent a two-stage surgical approach $(\mathrm{n}=8)$ and those not reimplanted with a TKA undergoing, rather, arthrodesis $(\mathrm{n}=3)$. The indication for one-stage revision was the diagnosis of PJI with a known causative organism. Two-stage revision was performed if the preoperative aspiration was culture-negative. An arthrodesis nail was implanted in cases of severe damage or rupture of the extensor mechanism. The diagnosis of PJI was based on an elevated preoperative C-reactive protein (CRP) level in combination with positive culture results of a preoperative aspiration after a minimum of 14 days of bacterial culture [7]. All patients were culture-positive and intraoperative sampling confirmed preoperative culture results (Table 1). There were no exclusions on the basis of patient condition, American Society of Anesthesiologists classification, body mass index, soft tissue status, including presence of a draining sinus, other patient-related factors potentially influencing the outcome [23], or a causative microorganism, including resistant organisms and fungi.

Of the 70 identified patients for our study, $11(16 \%)$ were lost to followup. Although several attempts to contact them were made, no records in the German federal medical database were found, and it could not be determined if these patients had died. Related general practitioners (family doctors) were unable to give further information; in addition, no clinical or radiologic data for the 11 patients were available. Fifty-nine patients comprised the final

Table 1. Microorganisms from preoperative cultures proven by intraoperative tissue sampling

\begin{tabular}{lll}
\hline Microorganism & Number & Percentage \\
\hline Staphylococcus epidermidis & 21 & 35.6 \\
MRSE & $(7)$ & $(11.9)$ \\
Other coagulase-negative staphylococci & 9 & 15.2 \\
Streptococcus spp & 7 & 11.9 \\
Staphylococcus aureus & 6 & 10.2 \\
MRSA & $(2)$ & $(3.4)$ \\
Enterobacter spp & 5 & 8.5 \\
Gram-negative bacteria & 3 & 5.1 \\
Enterococcus spp & 2 & 3.4 \\
Peptostreptococcus spp & 2 & 3.4 \\
Polymicrobial infections & 3 & 5.1 \\
Fungal infection & 1 & 1.7
\end{tabular}

Numbers in parentheses are part of the total number of the original organism above; MRSE $=$ methicillin-resistant $S$ epidermidis $;$ MRSA = methicillin-resistant $S$ aureus. 
study cohort; all included had complete clinical and radiologic data. A total of 13 patients died during the followup. In 46 patients, clinical evaluation, including radiographic followup, was available within the last year before the study. Average followup was 10 years (last followup 2012-2013; range, 9-11 years). The mean age of the study patients at the time of surgery was 70 years (range, 60-81 years); 31 patients were women and 39 were men.

All one-stage exchange procedures followed the same standardized surgical protocol. The previous skin scar was excised and subcutaneous exposure was meticulous to find possible stitch abscesses or old sutures. For all septic knee revisions in 2002, a subvastus approach, the traditional approach in our hospital at that time, was used. Extraarticular débridement of the joint capsule and the synovium was performed first to open the joint as late as possible. The joint was then opened and aggressive débridement was used to remove all infected soft tissue including a complete synovectomy. Our technique in 2002 included the excision of the collateral ligaments and the posterior cruciate ligament to completely expose the posterior capsule, which was excised as well. Presently the collaterals are detached and débrided rather than excised. Solidly fixed implants were explanted with osteotomes or small power saw blades. Intraoperative samples (five for culture, two for histopathology) were taken from the soft tissues and the implant interface, following which intravenous antibiotics specified by an infectious disease consultant were administered. The wound was then lavaged by low-pressure pulsatile lavage with 3000 to $6000 \mathrm{~mL}$ of $0.02 \%$ polyhexanid solution (Lavasept ${ }^{\circledR}$; B. Braun, Melsungen, Germany). The surgery site was then redraped and gowns, gloves, suction tip, light handles, and instruments were exchanged. Completely new prepping was not possible, because the wound was open. Reconstruction of the joint was carried out with implantation of a cemented rotatinghinge knee implant (Endo Model ${ }^{\circledR}$; W. Link, Hamburg, Germany; FDA-approved implant). No bone grafts were used because in septic revision, a higher risk of periprosthetic infection has been reported when using allografts [2, 17, 33]. Antibiotic-loaded polymethylmethacrylate (PMMA) bone cement was used for both the fixation of the new implant and reconstruction of bone defects. Therefore, the premixed gentamicin and clindamycin-loaded bone cement $\left(\mathrm{Copal}^{\circledR}{ }^{\circledR}\right.$; Heraeus Medical, Wehrheim, Germany) was mixed with a maximum of $2 \mathrm{~g}$ specific antibiotic powder per $40 \mathrm{~g}$ PMMA. In 32 patients $(54 \%)$ the antibiotics used were gentamicin and clindamycin; in nine patients vancomycin mixed with gentamicin; in five patients $(9 \%)$ vancomycin mixed with ofloxacin and gentamicin; in four patients $(6 \%)$ vancomycin and clindamycin; in four patients $(6 \%)$ vancomycin, ampicillin, and gentamicin; in two patients (4\%) vancomycin alone; in two patients $(3 \%)$ cefuroxime and gentamicin; and in another two patients clindamycin, gentamicin, and ofloxacin.

A primary multilayer soft tissue closure was achieved and drainage was inserted into the joint.

Wound drainage was removed 2 days after surgery and full weightbearing with crutches was allowed from the first day on. Change of dressing was done every second day. To achieve the best possible ROM, postoperative intensive physiotherapy was applied and sufficient analgesia according to the recommendation of the World Health Organization was given in all patients. Other specific modalities such as heat were not used and no braces were used. Subcutaneous enoxaparin was used for prophylaxis of venous thromboembolism in general.

All patients received local and systemic antibiotic therapy based on the recommendation of a designated infectious disease consultant, who also supervised the whole course of antibiotic treatment during hospitalization. The average duration of intravenous antibiotic treatment was 14.2 days (range, 10-17 days). Duration was dependent on wound healing and constantly decreasing serum laboratory infection parameters (CRP and white blood cell count). Prolonged oral antibiotic therapy was not administered.

All patients were characterized as either "infection controlled" or "failure." Criteria for successful infection control were defined as no clinical signs of infection, no further surgery with the diagnosis of PJI, and no further positive cultures after the one-stage septic exchange. Failures included patients with local or systemic symptoms of infection, those needing further surgery as a result of persistent PJI according to the Musculoskeletal Infection Society criteria, and patients who died after generalized sepsis [42]. We considered reinfection to be an infection of the same joint with the same or another organism [14]. Additionally, we considered aseptic failures of the prosthetic device to be any procedure in which a reoperation was performed for reasons unrelated to PJI or sepsis. The HSS score was measured pre- and postoperatively [31] at the most recent followup visit. Patient radiographs were reviewed at the most recent postoperative followup and compared with those taken in the immediate index postoperative period to look for signs of mechanical failure or aseptic loosening of the prosthetic components.

All data registered before the most recent followup were available through the clinical database. Clinical examination was done by one of the observers (AZ). Radiological examination was done in the form of plain radiographs (AP and lateral view) and evaluated by two of the authors (AZ, DOK). 


\section{Statistical Analysis}

A paired t-test was used for calculating the differences in the pre- and postoperative clinical results with a confidence interval (CI) of 95\%. Probability $<0.05$ was considered statistically significant. As a result of more than $15 \%$ of unrelated deaths during the followup, competing risks survival analysis was performed. The uncertainty of the fractional survival was considered as a standard error or 95\% CIs. Standard errors are calculated by the method of Greenwood by using GraphPad for Windows (GraphPad Software, La Jolla, CA, USA).

\section{Results}

At the end of followup, five patients were rated as reinfected. Two of these patients died because of systemic sepsis; the affecting organism is not known. In one of the patients, the same Staphylococcus epidermidis organism was found at the revision surgery. The two other patients developed a fungal periprosthetic infection with Candida parapsilosis. In the three patients with a reinfection, a second one-stage exchange was performed, whereby one of these 2 years later sustained a third periprosthetic infection, then after another one-stage exchange, there was no recurrent infection until the latest followup. The competing risk survival analysis revealed $93 \%$ with a mean of 4.1 and a $95 \%$ CI of $89 \%$ to $96 \%$ at 10 years for patient survival free of infection $(\mathrm{p}<0.007)$.

Most infections $(\mathrm{n}=21)$ were caused by $S$ epidermidis followed by nine patients $(15.2 \%)$ with other Staphylococcus, in seven (11.9\%) Streptococcus, and in six (10.2\%) Staphylococcus aureus. If we hypothesize a critical "worst case scenario" where all 11 patients lost to followup experienced reinfection, then only 54 of 70 (77\%) would have been infection-free.

After the end of followup there were seven patients with need for revision surgery because of aseptic loosening. In three patients there was a sole loosening of the tibia and in four patients of the tibial and femoral component. Competing risk survival analysis revealed $91 \%$ survival at 10 years with a mean of 5.2 and a $95 \%$ CI of $86 \%$ to $95 \%$ for patient survival free of reoperation for aseptic loosening reasons $(\mathrm{p}<0.002)$. After 10 years there were 25 patients left at risk for aseptic loosening. The 10-year survivorship free of any reoperation, including both septic and aseptic causes, was $75 \%$ (95\% CI, 60\%-87\%).

The HSS score improved significantly from a mean preoperative value of 35 ( $\pm 24.2 \mathrm{SD}$; range, 13-99) to an average of 69.6 ( $\pm 22.5 \mathrm{SD}$; range, 22-100) at latest followup. Postoperative excellent results (100 to 85 points) for HSS score were found in 30\%, good results (84-70 points) in $33 \%$, fair (69 to 60 points) in $11 \%$, and poor ( $<60$ points) in $26 \%$. The flexion of the knee improved significantly from a mean value of $50^{\circ}( \pm 25.6 \mathrm{SD}$; range, $8-144)$ to $76^{\circ}$ ( $\pm 33 \mathrm{SD}$; range, 8-144). The results for pain (maximum of 30 points) were a mean preoperative 5.7 ( \pm 9.9; range, 0-30) and improved significantly postoperatively to 18.5 points ( \pm 11.3 ; range, $0-30)$. A significant improvement was also found regarding the function preand postoperatively with a mean 10 points preoperatively ( \pm 6; range, 4-22) and 16.7 ( \pm 6.3 ; range, 4-22) postoperatively.

In addition to the six patients who underwent revision for aseptic loosening, six other patients showed radiolucent lines in the area of the metaphysis of the tibia and femur. Because the radiolucencies were less than $2 \mathrm{~mm}$ in thickness and nonprogressive, no further assessment was recommended.

\section{Discussion}

Infection rates associated with primary TKA are reported to be between $0.6 \%$ and $1.77 \%[21,22]$. The major treatment options for chronic PJI consist of two-stage revision with an interval of antibiotic-loaded PMMA spacer and antibiotic management and one-stage revision with direct exchange of the implants using antibiotic-loaded bone cement for fixation [43]. In this study our intention was to demonstrate our results of one-stage exchange to a rotating hinge device using a strict protocol and limited exclusion criteria for the patients. We specifically examined infection-free survival, survival free of reoperation for aseptic causes, functional improvement measured by HSS score, and radiographic evidence of loosening or implant migration.

In our study there were some clear limitations and assumptions. The followup was long enough to clearly identify recurrent or residual infection and early prosthetic failures, but may not have been long enough to identify long-term issues with a highly constrained device. The analysis was retrospective with no matching control group, and some data that might have been prospectively gathered were unavailable. There was also a high percentage (15.7\%) of patients lost to followup; it is possible that some of these patients might have developed recurrent infection or loosening. Several attempts were made to contact these patients but no actual information was possible to obtain from the individuals or their families. The number of patients who died after surgery during the 10-year observation period should be viewed from the perspective of the perioperative mortality rate of any type of septic revision [29] and with the average age of the patients at surgery (69.4 years) taken into account. Another limitation of the 
current study is the definition of "infection-free." Our definition was based on clinical signs of PJI and not on aspiration or serologic testing at the time of latest followup, which was judged to be unreasonable in asymptomatic patients. Infection control after TKA is influenced by several risk factors, including patient comorbidities, sex, and the causative microorganism [14, 40]. A powerful statistical analysis of these factors was not possible as a result of the low number of patients in each group with risk factors. A final limitation was our chosen clinical assessment tool, the HSS score, because many other one-stage exchange studies have used the Knee Society score.

Our results in terms of survivorship free from infection with the single-stage approach appear comparable to those offered by a systematic review of the results of septic TKA revision, which reported mean eradication rates of $90.6 \%$ for two-stage and $89.2 \%$ for one-stage surgery [32] as well as with other recent studies on the subject of one-stage exchange [36, 39, 40]. The higher eradication rate after the two-stage revision, especially in combination with a mobile spacer, was based on numerous publications with shortterm followup and/or a low number of study patients with an eradication rate of up to $100 \%$. In studies with comparable numbers and followups to our own, eradication rates ranged between $87.8 \%$ and $91.7 \%$ (Tables 2, 3). Better results can be achieved by following strict inclusion criteria, choosing the one-stage approach only for highly selected patients with intact soft tissues, and susceptible microorganisms [14]. Standardized diagnostic and therapeutic algorithms for one-stage exchange have been described to make multicenter studies possible with comparable cohorts [13]. Two-stage revision with mobile or static spacers requires at least two surgeries, a longer period of time with limited mobility in the interval, and more possibility for perioperative complications and morbidity [24, 28, 32, 39].

Seven patients $(11.9 \%)$ had to be revised as a result of aseptic complications after our one-stage exchange; there were six instances of aseptic loosening with revision of at least one component, and one patient was revised as a result of a periprosthetic fracture. The average time to loosening necessitating revision was 5.1 years (range, 2-8 years) after the index one-stage exchange arthroplasty.

The reported rate of aseptic loosening of a hinged knee prosthesis is quite variable. Gehrke et al. [9] found a survival rate of $90 \%$ in 141 patients at 13 years in primary TKA with excellent and good results for the HSS score in $64 \%$ of the patients (100 to 70 points) with the same

Table 2. Publications reporting results of one-stage septic exchange after TKA

\begin{tabular}{|c|c|c|c|c|c|}
\hline Study & Journal & Year of publication & TKA & Followup (years) & Eradication rate $(\%)$ \\
\hline Freeman et al. [8] & $J$ Bone Joint Surg Br & 1985 & 8 & 2.2 & 100 \\
\hline von Foerster et al. [41] & Orthopäde & 1991 & 104 & NA & 73.1 \\
\hline Goksan and Freeman [10] & $J$ Bone Joint Surg Br & 1992 & 18 & 5 & 89 \\
\hline Lu et al. [25] & Zhonghua Wai Ke Za Zhi & 1997 & 8 & 1.7 & 100 \\
\hline Silva et al. [34] & Clin Orthop Relat Res & 2002 & 37 & 4 & 89.2 \\
\hline Buechel et al. [5] & Am J Orthop (Belle Mead NJ) & 2004 & 21 & 10.2 & 90.9 \\
\hline Sofer et al. [37] & Orthopäde & 2005 & 15 & 1.5 & 93.3 \\
\hline Bauer et al. [1] & Rev Chir Orthop Reparatrice Appar Mot & 2006 & 30 & 4.3 & NA \\
\hline Singer et al. [36] & Clin Orthop Relat Res & 2012 & 63 & 3 & 95 \\
\hline Jenny et al. [20] & Clin Orthop Relat Res & 2013 & 47 & 2.75 & 87 \\
\hline Haddad et al. [14] & Clin Orthop Relat Res & 2015 & 28 & 6.5 & 100 \\
\hline Tibrewal et al. [39] & Bone Joint J & 2014 & 50 & 10.5 & 98 \\
\hline Current study & & & 70 & 10 & 93.0 \\
\hline
\end{tabular}

$\mathrm{NA}=$ not available.

Table 3. Published study results of two-stage exchange arthroplasty with long followup and/or a larger study cohort

\begin{tabular}{|c|c|c|c|c|c|}
\hline Study & Journal & Year of publication & Followup (years) & TKA & Eradication rate $(\%)$ \\
\hline Goldman et al. [11] & Clin Orthop Relat Res & 1996 & 7.5 & 64 & 90.6 \\
\hline Emerson et al. [6] & Clin Orthop Relat Res & 2002 & 7.5 & 48 & 91.7 \\
\hline Haleem et al. [15] & Clin Orthop Relat Res & 2004 & 7.2 & 96 & 90.6 \\
\hline Hsu et al. [18] & $J$ Arthroplasty & 2007 & 8.4 & 28 & 89.3 \\
\hline Gooding et al. [12] & Clin Orthop Relat Res & 2011 & 9.0 & 115 & 87.8 \\
\hline
\end{tabular}


implant used in this study. Bistolfi et al. [4] found a reoperation-free survival rate of $79 \%$ in 32 patients with a hinged TKA used for revision TKA after a mean of $155 \pm$ 40.1 months followup. The main reason for revision was aseptic loosening in four patients. There is no literature to date that reports the number of revisions resulting from aseptic loosening of a hinged prosthesis after septic onestage exchange.

We found a significantly improved HSS score from a mean preoperative value of 35 ( $\pm 24.2 \mathrm{SD}$; range, 13-99) to an average of $69.6( \pm 22.5 \mathrm{SD}$; range, $22-100)$ at latest followup. Postoperative excellent results for HSS score were found in $30 \%$ (100 to 85 points), good results in $33 \%$ ( 84 to 70 points), fair in $11 \%$ (69 to 60 points), and poor in $26 \%$ ( $<60$ points). Tibrewal et al. [39] performed 50 onestage exchanges in infected TKAs and found significant improvement in the Oxford Knee Score of 20.0 points (95\% CI, 17.8-22.2; p < 0.001) 1 year after surgery. In another study 28 patients with one-stage exchange were compared with 74 patients with two-stage exchange. The single-stage group had a significantly higher Knee Society score at 2 years than the two-stage group (mean, 88; range, 38-97 versus 76; range, 29-93; $p=0.001$ ). However, only "easy" infected cases ("easy" considered having good soft tissue, no bone loss, no sinus germs, and no multiresistant germ as a pathogen) were included in the one-stage group. Similar results were found by Jenny et al. [20] in 42 patients after one-stage exchange. At latest followup, the median Knee Society knee score was 85 points (range, $10-100$ points), the median Knee Society pain score was 45 points (range, 10-50 points), and the median Knee Society function score was 78 points (range, 0-100 points). Although the Knee Society score is not directly comparable to the HSS score, the authors feel these results are in line with other one-stage exchange results with comparable good and excellent results in scores in $63 \%$. The flexion angle within mean $76^{\circ}$ ( $\pm 33 \mathrm{SD}$; range, 8-144; median, $88^{\circ}$ ) seems after a 7 -year longer followup an acceptable result.

After a 10-year followup, in only 12 patients were radiolucent lines visible; in six of these patients, revision surgery for aseptic loosening was necessary. There are no explicit data available regarding one-stage exchange and loosening of rotating hinge prostheses in this special situation. However, a higher aseptic loosening rate is expected in hinged prostheses because the increased constraint transfers stress to the bone-implant interface; rotating hinge devices may moderate this effect [38].

Key factors for the successful treatment of PJI in TKA are the preoperative diagnostic approach, known susceptibility of the causative microorganism, aggressive débridement after a standardized surgical protocol, and the combination of local and systemic antibiotic therapy.
Based on a systematic review of the current literature, no direct comparative studies demonstrate the clear superiority of either the one-stage or two-stage exchange method [19]. Our results suggest that there is indeed a role for onestage exchange, and both our study and others [14] emphasize this. Further studies with a multicenter prospective, comparative approach are needed to validate our results and to compare the two methods more directly.

\section{References}

1. Bauer T, Piriou P, Lhotellier L, Leclerc P, Mamoudy P, LortatJacob A. [Results of reimplantation for infected total knee arthroplasty: 107 cases] [in French]. Rev Chir Orthop Reparatrice Appar Mot. 2006;92:692-700.

2. Bauman RD, Lewallen DG, Hanssen AD. Limitations of structural allograft in revision total knee arthroplasty. Clin Orthop Relat Res. 2009;467:818-824.

3. Berend KR, Lombardi AV Jr, Morris MJ, Bergeson AG, Adams JB, Sneller MA. Two-stage treatment of hip periprosthetic joint infection is associated with a high rate of infection control but high mortality. Clin Orthop Relat Res. 2013;471:510-518.

4. Bistolfi A, Rosso F, Crova M, Massazza G. Endo-Modell rotating-hinge total knee for revision total knee arthroplasty. Orthopedics. 2013;36:1299-1306.

5. Buechel FF, Femino FP, D'Alessio J. Primary exchange revision arthroplasty for infected total knee replacement: a long-term study. Am J Orthop (Belle Mead NJ). 2004;33:190-198.

6. Emerson RH Jr, Muncie M, Tarbox TR, Higgins LL. Comparison of a static with a mobile spacer in total knee infection. Clin Orthop Relat Res. 2002;404:132-138.

7. Fink B, Makowiak C, Fuerst M, Berger I, Schäfer P, Frommelt L. The value of synovial biopsy, joint aspiration and C-reactive protein in the diagnosis of late peri-prosthetic infection of total knee replacements. J Bone Joint Surg Br. 2008;90:874-878.

8. Freeman MA, Sudlow RA, Casewell MW, Radcliff SS. The management of infected total knee replacements. J Bone Joint Surg Br. 1985;67:764-768.

9. Gehrke T, Kendoff D, Haasper C. The role of hinges in primary knee arthroplasty. Bone Joint J. 2014;96(Suppl A):93-95.

10. Goksan SB, Freeman MA. One-stage reimplantation for infected total knee arthroplasty. J Bone Joint Surg Br. 1992;74:78-82.

11. Goldman RT, Scuderi GR, Insall JN. 2-stage reimplantation for infected total knee replacement. Clin Orthop Relat Res. 1996;331:118-124.

12. Gooding CR, Masri BA, Greidanus NV, Garbuz DS. Durable infection control and function with the PROSTALAC spacer in two-stage revision for infected knee arthroplasty. Clin Orthop Relat Res. 2011;469:985-993.

13. Gulhane S, Vanhegan IS, Haddad FS. Single stage revision: regaining momentum. J Bone Joint Surg Br. 2012;94(Suppl A): $120-122$.

14. Haddad FS, Sukeik M, Alazzawi S. Is single-stage revision according to a strict protocol effective in treatment of chronic knee arthroplasty infections? Clin Orthop Relat Res. 2015;473:8-14.

15. Haleem AA, Berry DJ, Hanssen AD. Mid-term to long-term followup of two-stage reimplantation for infected total knee arthroplasty. Clin Orthop Relat Res. 2004;428:35-39.

16. Herbert CK, Williams RE, Levy RS, Barrack RL. Cost of treating an infected total knee replacement. Clin Orthop Relat Res. 1994;331:140-145. 
17. Hilgen V, Citak M, Vettorazzi E, Haasper C, Day K, Amling M, Gehrke T, Gebauer M. 10-year results following impaction bone grafting of major bone defects in 29 rotational and hinged knee revision arthroplasties: a follow-up of a previous report. Acta Orthop. 2013;84:387-391.

18. Hsu YC, Cheng HC, Ng TP, Chiu KY. Antibiotic-loaded cement articulating spacer for 2-stage reimplantation in infected total knee arthroplasty. A simple and economic method. J Arthroplasty. 2007;22:1060-1066.

19. Jämsen E, Stogiannidis I, Malmivaara A, Pajamäki J, Puolakka T, Konttinen YT. Outcome of prosthesis exchange for infected knee arthroplasty: the effect of treatment approach. Acta Orthop. 2009;80:67-77.

20. Jenny JY, Barbe B, Gaudias J, Boeri C, Argenson JN. High infection control rate and function after routine one-stage exchange for chronically infected TKA. Clin Orthop Relat Res. 2013;471:238-243.

21. Joulie D, Girard J, Mares O Beltrand E, Legout L, Dezèque H, Migaud H, Senneville E. Factors governing the healing of Staphylococcus aureus infections following hip and knee prosthesis implantation: a retrospective study of 95 patients. Orthop Traumatol Surg Res. 2011;97:685-692.

22. Kurtz SM, Ong KL, Lau E, Bozic KJ, Berry D, Parvizi J. Prosthetic joint infection risk after TKA in the Medicare population. Clin Orthop Relat Res. 2010;468:52-56.

23. Laffer RR, Graber P, Ochsner PE, Zimmerli W. Outcome of prosthetic knee-associated infection: evaluation of 40 consecutive episodes at a single centre. Clin Microbiol Infect. 2006;12: 433-439.

24. Leone JM, Hanssen AD. Management of infection at the site of a total knee arthroplasty. J Bone Joint Surg Am. 2005;87:2335-2348.

25. Lu H, Kou B, Lin J. [One-stage reimplantation for the salvage of total knee arthroplasty complicated by infection] [in Chinese]. Zhonghua Wai Ke Za Zhi. 1997;35:456-458.

26. Masters JPM, Smith NA, Foguet P, Reed M, Parsons H, Sprowson AP. A systemic review of the evidence for single stage and two stage revision of infected knee replacement. BMC Musculoskelet Disord. 2013;14:222-234.

27. Mortazavi SM, Vegari D, Ho A, Zmistowski B, Parvizi J. Twostage exchange arthroplasty for infected total knee arthroplasty: predictors of failure. Clin Orthop Relat Res. 2011;469: 3049-3054.

28. Parkinson RW, Kay PR, Rawal A. A case for one-stage revision in infected total knee arthroplasty? Knee. 2011;18:1-4.

29. Parvizi J, Azzam K, Ghanem E, Austin MS, Rothman RH. Periprosthetic infection due to resistant staphylococci: serious problems on the horizon. Clin Orthop Relat Res. 2009;467: $1732-1739$.
30. Pitto RP, Castelli CC, Ferrari R, Munro J. Pre-formed articulating knee spacer in two-stage revision for the infected total knee arthroplasty. Int Orthop. 2005;29:305-308.

31. Ranawat CS, Shine JJ. Duo-condylar total knee arthroplasty. Clin Orthop Relat Res. 1973;94:185-195.

32. Romanò CL, Gala L, Logoluso N, Romanò D, Drago L. Twostage revision of septic knee prosthesis with articulating knee spacers yields better infection eradication rate than one-stage or two-stage revision with static spacers. Knee Surg Sports Traumatol Arthrosc. 2012;20:2445-2453.

33. Schmitz HC, Klauser W, Citak M, Al-Khateeb H, Gehrke T, Kendoff D. Three-year follow up utilizing tantal cones in revision total knee arthroplasty. J Arthroplasty. 2013;28:1556-1560.

34. Silva M, Tharani R, Schmalzried TP. Results of direct exchange or debridement of the infected total knee arthroplasty. Clin Orthop Relat Res. 2002;404:125-131.

35. Silvestre A, Almeida F, Renovell P, Morante E, López R. Revision of infected total knee arthroplasty: two-stage reimplantation using an antibiotic-impregnated static spacer. Clin Orthop Surg. 2013;5:180-187.

36. Singer J, Merz A, Frommelt L, Fink B. High rate of infection control with one-stage revision of septic knee prostheses excluding MRSA and MRSE. Clin Orthop Relat Res. 2012;470:1461-1471.

37. Sofer D, Regenbrecht B, Pfeil J. [Early results of one-stage septic revision arthroplasties with antibiotic-laden cement. A clinical and statistical analysis] [in German]. Orthopäde. 2005;34:592-602.

38. Springer BD, Hanssen AD, Sim FH, Lewallen DG. The kinematic rotating hinge prosthesis for complex knee arthroplasty. Clin Orthop Relat Res. 2001;392:283-291.

39. Tibrewal S, Malagelada F, Jeyaseelan L, Posch F, Scott G. Single-stage revision for the infected total knee replacement. Results from a single centre. Bone Joint J. 2014;96:759-764.

40. Vanhegan IS, Morgan-Jones R, Barrett DS, Haddad FS. Developing a strategy to treat established infection in total knee replacement. A review of the latest evidence and clinical practice. J Bone Joint Surg Br. 2012;94:875-881.

41. von Foerster G, Kluber D, Kabler U. [Mid- to long-term results after treatment of 118 cases of periprosthetic infections after knee joint replacement using one-stage exchange surgery] [in German]. Orthopäde. 1991;20:244-252.

42. Workgroup Convened by the Musculoskeletal Infection Society. New definition for periprosthetic joint infection. J Arthroplasty. 2011;26:1136-1138

43. Zimmerli W, Trampuz A, Ochsner PE. Prosthetic-joint infections. $N$ Engl J Med. 2004;351:1645-1654.

44. Zmistowski B, Karam JA, Durinka JB, Casper DS, Parvizi J. Periprosthetic joint infection increases the risk of one-year mortality. J Bone Joint Surg Am. 2013;95:2177-2184. 